\title{
A Case of Severe Thyroid Eye Disease Treated with Tocilizumab
}

\author{
Aysel Mehmet ${ }^{1, *}$, Eirini Kanella Panagiotopoulou ${ }^{1}$, Aristeidis Konstantinidis ${ }^{1}$, Charalampos Papagoras ${ }^{2}$, \\ Panagiotis Skendros ${ }^{2}$, Doukas Dardabounis ${ }^{1}$, Athanasia Maria Mikropoulou ${ }^{1}$, Georgios Labiris ${ }^{1}$
}

\begin{abstract}
This is a case report describing a patient with severe thyroid eye disease complicated with dysthyroid optic neuropathy that was unresponsive to intravenous steroids and orbital radiotherapy but responded well to intravenous tocilizumab.

KEYWORDS

thyroid eye disease; tocilizumab

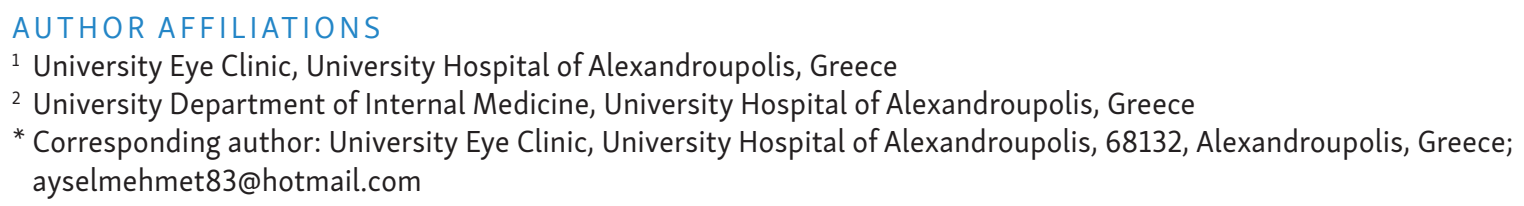

Received: 10 November 2020

Accepted: 18 January 2021

Published online: 14 April 2021

Acta Medica (Hradec Králové) 2021; 64(1): 64-69

https://doi.org/10.14712/18059694.2021.12

(c) 2021 The Authors. This is an open-access article distributed under the terms of the Creative Commons Attribution License (http://creativecommons.org/licenses/by/4.0), which permits unrestricted use, distribution, and reproduction in any medium, provided the original author and source are credited. 


\section{INTRODUCTION}

Thyroid eye disease (TED) is an inflammatory disorder of the orbit secondary to Graves' disease. Although the inflammatory phase is self-limiting it can severely affect the vision. Sight threatening TED is rare. The overall incidence of TED is $0.42 / 10,000 /$ year with women and the $40-60$ age group being mostly targeted by the disease (1). Only about $2 \%$ of patients with TED have complications that threaten the vision primarily due to optic neuropathy and/or corneal exposure.

Cessation of smoking is the most important lifestyle factor that positively influences TED; while systemic steroids is the first line medication in the case of moderate-to-severe and sight threatening TED (1). However poor or no response to steroids is occasionally encountered (2). In these cases, steroid administration could be attempted again; or proceed to cyclosporine or rituximab administration and/or orbital radiotherapy. Tocilizumab is a recombinant antihuman monoclonal antibody of the immunoglobulin G1 subclass directed against the interleukin-6 receptor (IL-6R) that has been used in TED patients with variable efficacy. Several theories have been proposed regarding tocilizumab's potential beneficial impact in TED. Among them, an overall modification of the immunomodulatory function, remodeling of the extracellular matrix, and reduction of the inflammatory response (3).

Within this context, we present a case with sight-threatening TED with minimal response to steroids and orbital radiotherapy, which responded well to tocilizumab treatment.

\section{CASE DESCRIPTION}

A 60-year-old man, heavy smoker (40 cigarettes/day, 80 pack/years), was referred to our outpatients' service by his doctor in February 2019 due to sight-threatening TED, which manifested with gradual reduction of his visual acuity. He was diagnosed with Graves' hyperthyroidism 14 years earlier, and ever since he was under regular systemic treatment with carbimazole. The patient had also had $I^{131}$ treatment in July 2018.

On the initial examination his best corrected visual acuities (BCVA) were 0.4 logMAR in the right eye (OD) and 0.3 logMAR in the left eye (OS) and the Clinical Activity Score (CAS) was 5/7. The exophthalmometry measurements were $32 \mathrm{~mm}$ OD and $30 \mathrm{~mm}$ OS. The intraocular pressures (measured with Goldmann Applanation Tonometry-GAT IOPs) were $30 \mathrm{mmHg}$ in both eyes in the primary gaze and $35 \mathrm{mmHg}$ in the upgaze. The right upper eyelid was retracted $2 \mathrm{~mm}$ above the limbus and the left was $0.5 \mathrm{~mm}$ above the limbus. Both lower lids were retracted $1.5 \mathrm{~mm}$ below the limbus (Fig. 1 above). The patient was hypermetropic (+3 sph dpt spherical equivalent) and the angles were moderately closed in the superior quadrants and moderately open in the inferior quadrants and gave a plateau iris configuration on indentation gonioscopy. Fundoscopy showed early disc swelling in the OD and increased retinal nerve fiber thickness bilaterally (Fig. 2). Patient was advised to quit smoking and a fixed
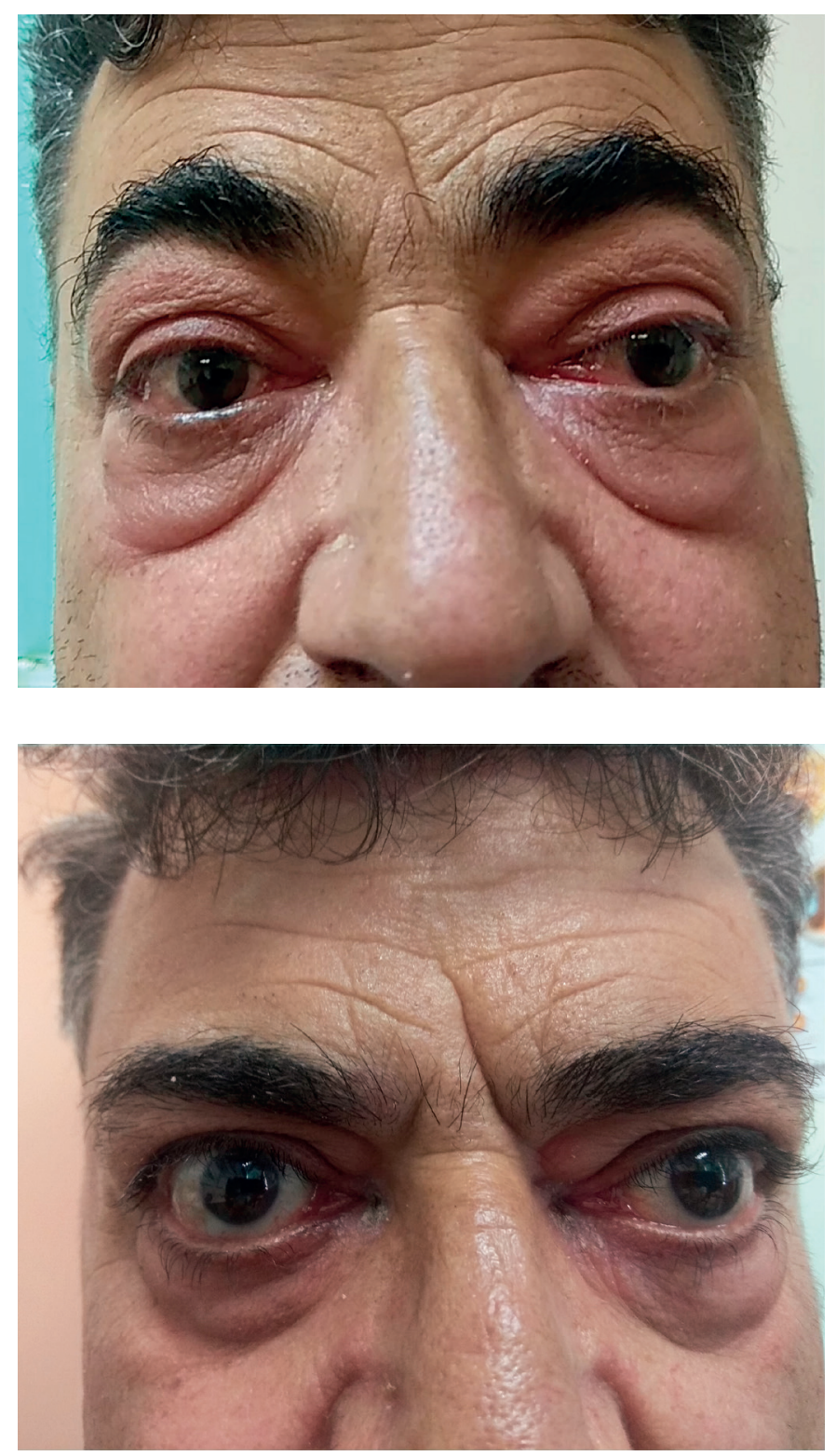

Fig. 1 Top: Baseline clinical picture. Upper and lower lid erythema and swelling of both eyes. Below: clinical picture after the 4-week course of tocilizumab. Marked reduction of erythema and swelling of all eyelids are noted.

combination of dorzolamide-timolol (FCDT) bid was immediately started. Automated perimetry showed defects bilaterally (Fig. 3a).

In order to address the increased intraorbital pressure, the patient was scheduled immediately for a 3-day course of intravenous (iv) methylprednisolone $1000 \mathrm{mg} /$ day. However, neither BCVA nor exophthalmos was improved. Orbital decompression was suggested but was rejected by the patient who was against any surgical intervention. As a second option, a 12-week course of intravenous methylprednisolone was proposed (500 mg/week for the first six weeks and then $250 \mathrm{mg} /$ week for the following six weeks) as per EUGOGO recommendations (1) aiming to achieve a prolonged anti-inflammatory effect. At the end of the course the CAS score was $8 / 10$ with no clinical improvement. The patient complained that his eyesight was worse and his BCVA was 0.5 logMAR OD and 0.4 logMAR OS. 
Nerve Fiber ONH/GCC Change Analysis

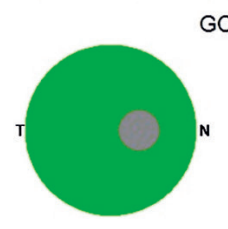

Visit Date: 02/18/19(59.1) SSI $=67$ (GCC) $/ 69(0)$

Visit Date: $07 / 24 / 19$ (59.6) SSI = N/A / 41 (ONH)

RNFL Analysis
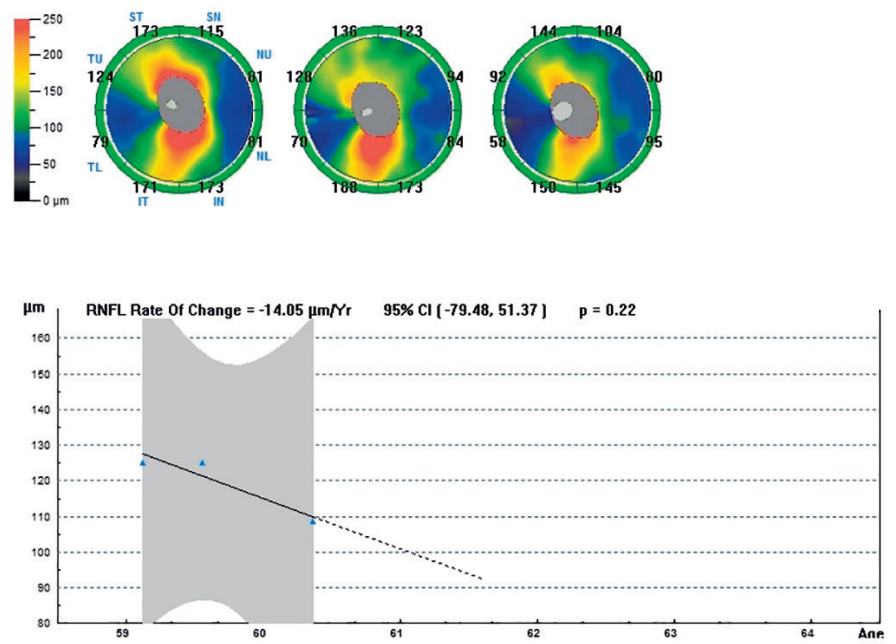

Nerve Fiber ONH/GCC Change Analysis

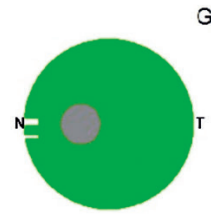

Visit Date: 02/18/19 (59.1)

Visit Date: 07/24/19 (59.6) SSI = N/A /34 (ONH)

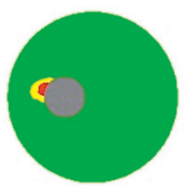

Visit Date: $05 / 13 / 20(60.4)$ $\mathrm{SSI}=54(\mathrm{GCO} / 35$ (ONH)

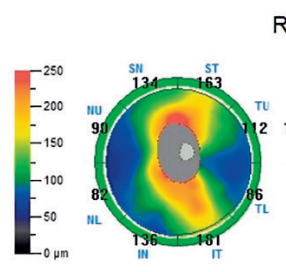

RNFL Analysis
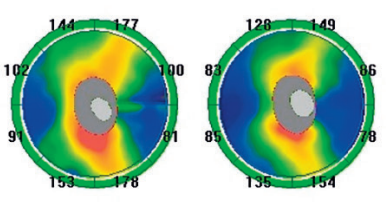

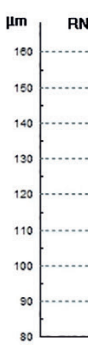

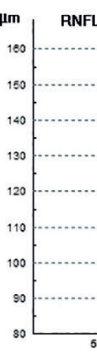

- NDB $\quad$ Thickness

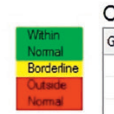
Comparison to NDB

Average $\mathrm{GCC}(\mu \mathrm{m})$

Superior GCC ( $(\mu \mathrm{m})$

Inferior $6 \subset C C(\mu \mathrm{m})$

FLV (\%)

GLV (\%)

RNFL/ONH Analysis Average RNFL ( $(\mu \mathrm{m})$ Superior RNFL ( $\mathrm{jm})$ Inferior RNFL ( $(\mathrm{m})$ Cup/Disc H. Ratio Cup/Disc V. Ratio Rim Area (mm')

Right / OD
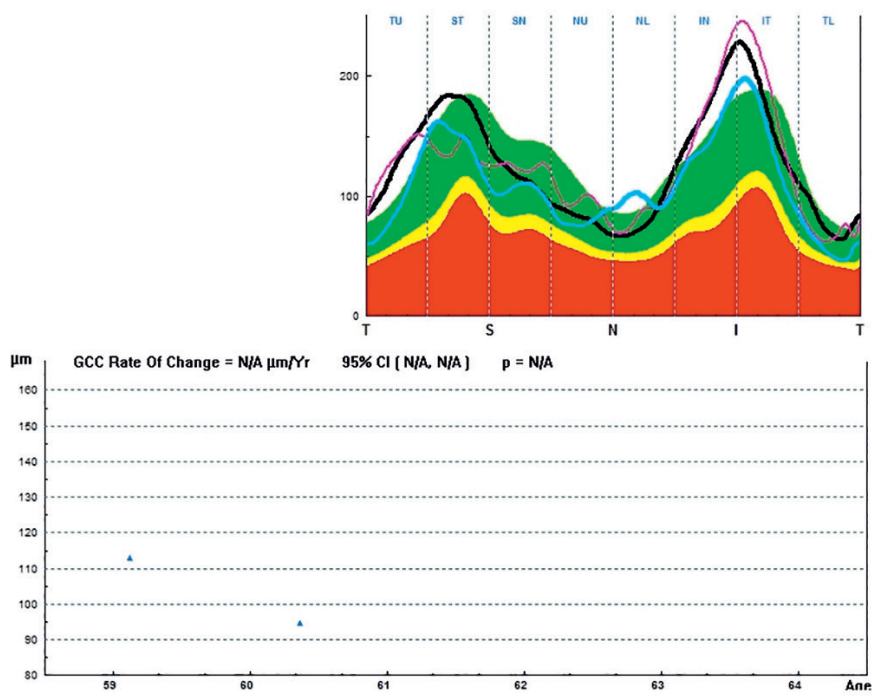

Left / OS

- NDB C Thickness

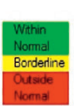

Comparison to NDB

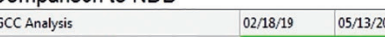

Average $6 C C(\mathrm{um})$ Superior GCC ( $(\mu \mathrm{m})$ Inferior $\operatorname{GCC}(\mu \mathrm{m})$ FLV (\%)

GLV (\%)

RNFL/ONH Anelysis Average RNFL ( $\mu \mathrm{m})$ Superior RNFL ( $(\mu \mathrm{m})$ Inferior RNFL ( $\mu \mathrm{m})$ Cup/Disc H. Ratio Cup/ /isc V. Ratio

- 05/13/20 Rim Area (mm)
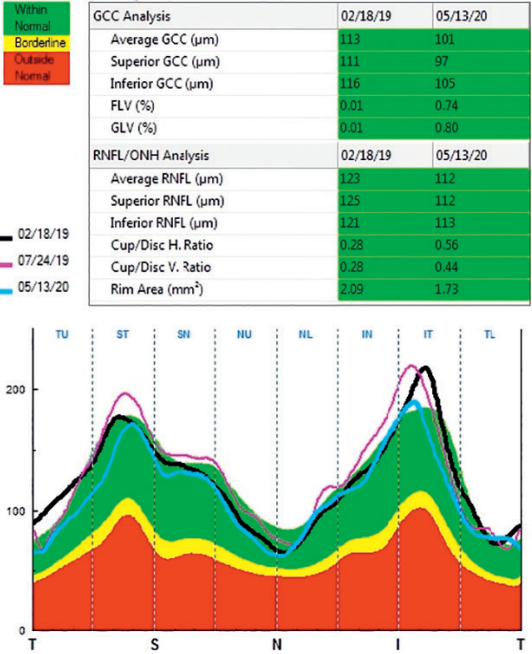

95\% Cl [ N/A, N/A $] \quad p=N / A$

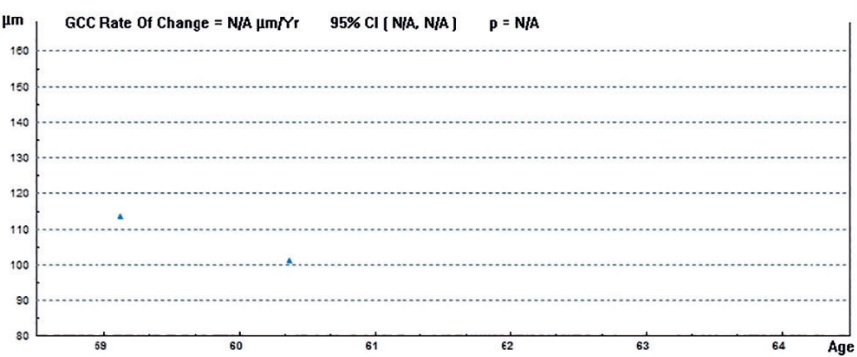

Fig. 2 Sequential measurements of the retinal nerve fiber layer of the right and left eye. 01/18/19: baseline OCT (black line), 7/24/19 OCT taken after the 12-week course of iv methylprednisolone (purple line), 5/13/20 OCT taken after the 4-week course of tocilizumab (light blue line). A reduction of the thickness of the retinal nerve fiber layer is shown (when comparing the height of the black line to the height of the light blue line). 
GAT-IOPs were $27 \mathrm{mmHg}$ in both eyes despite the FCDT, with deterioration of his visual fields (Fig. 3b). The patient consented to orbital radiotherapy and had 10 sessions with a cumulative dose of $20 \mathrm{~Gy}$. Despite the treatment there was no improvement in BCVA, GAT-IOPs or visual fields (Fig. 3c). One month after the orbital radiotherapy he underwent thyroidectomy.

As the postoperative period was uneventful and in order to address the persisting sight-threatening situation we decided to start the patient on intravenous tocilizumab (RoACTEMRA, Roche International Ltd, Walwyn Garden City, UK), initially one injection every 4 weeks at a dose of $8 \mathrm{mg} / \mathrm{ml}$. After the fourth infusion (one month after the first tocilizumab infusion) the patient had a marked improvement of his BCVA (0.2 logMAR OD, 0.0 logMAR OS), reduction of the IOP $(20 \mathrm{mmHg}$ bilaterally in primary position, $23 \mathrm{mmHg}$ in upgaze), slight improvement of the lid retraction (the right upper eyelid was $1 \mathrm{~mm}$ above the limbus, the left upper eyelid $0.5 \mathrm{~mm}$ below the limbus but both lower lids remained $1.5 \mathrm{~mm}$ below the limbus), the range of ocular movements was extended and the visual fields were also improved (Fig. 3d). The exophthalmometry measurements remained the same (Fig. 1 below). The retinal nerve fiber thickness was also reduced bilaterally (Fig. 2). The patient had only managed to reduce smoking to $20 \mathrm{ci}-$ garettes/day. He was on a dietary supplement of selenium, topical artificial tears and the FCDT. Three months the course of tocilizumab all patient's indices remain stable.

\section{DISCUSSION}

Thyroid eye disease can potentially cause blindness in a small minority of patients. The mainstay of treatment for the moderate-to-severe disease is a 12-week course of intravenous steroids and for the sight threatening form a 3-day course of high-dose intravenous steroids and/or orbital decompression (1).

Intravenous steroids have been associated with recurrences and poor response as well as $6.5 \%$ morbidity and $0.5 \%$ mortality rate (4). For this reason other modalities have been tried such as cyclosporine, azathioprine, a

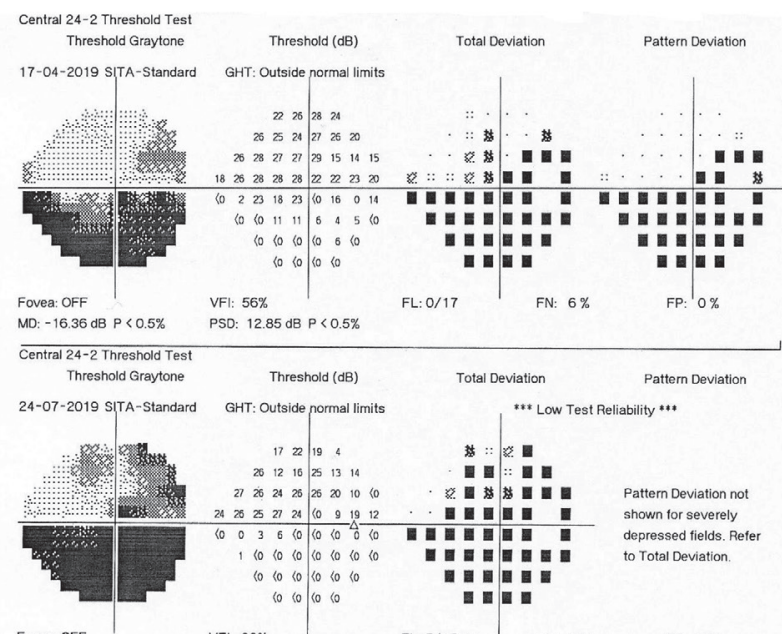

b

C

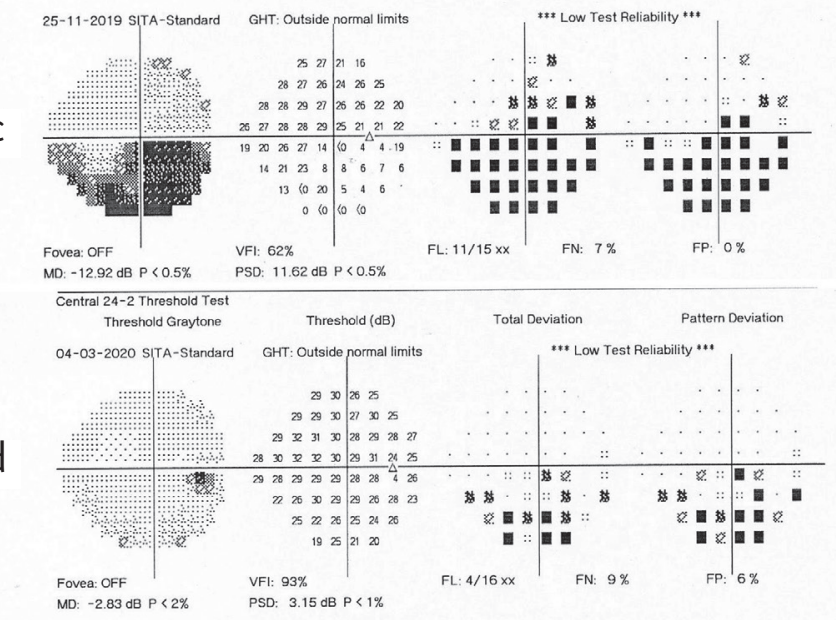

a
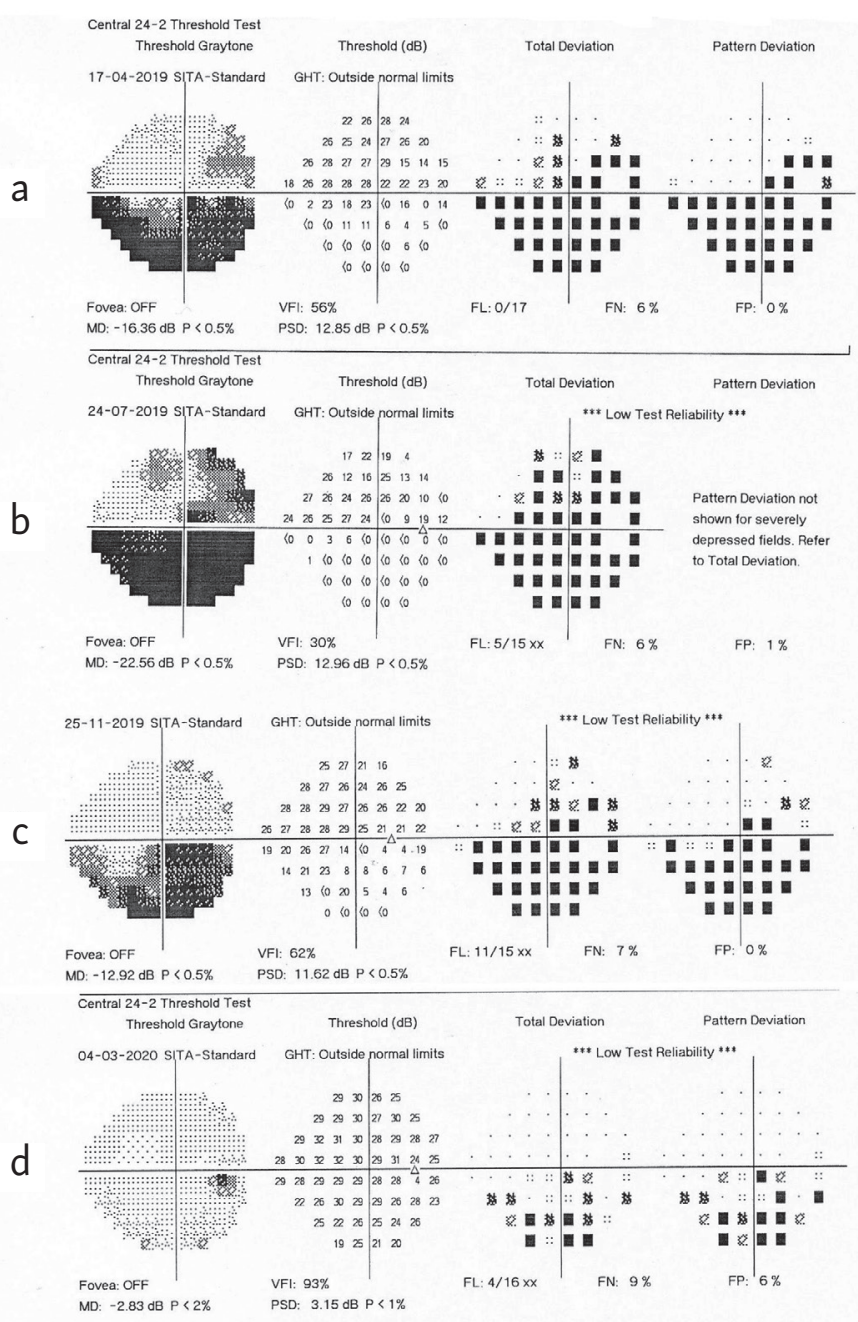

Fig. 3 Serial visual fields of the right eye (left) and left eye (right). total deviation and pattern deviation plots are only shown. (a) Baseline VF. Right eye: superior paracentral scotoma with dense inferior altitudinal scotoma. Left eye: Dense inferior altitudinal scotoma; (b) VFs after the 12-week course of intravenous methylprednisolone. There is no change in the visual field defects; (c) VFs after radiotherapy. There is no change in the visual fields defects; (d) VFs after the 4-week course of tocilizumab. Right eye: the superior paracentral scotoma has disappeared and the inferior altitudinal defect has decreased in depth (sensitivity has increased) and size. Left eye: The inferior altitudinal scotoma has decreased in depth (sensitivity has increased) and size. 
mycophenolate mofetil, methotrexate along with specific immunosuppresants (rituximab, adalimumab, etanercept, infliximab) (5). These treatments are reserved for the moderate-to-severe TED. In the case of sight threatening TED more radical measures are required.

Radiotherapy has been used in the treatment of TED for many decades. It mainly acts on the orbital soft tissues and the extraocular muscles (6). Its effects are more potent and last longer when combined with steroids (oral or iv) $(6,7)$. The effects of radiotherapy may take days to a few weeks to develop (1). Given this time frame and bearing in mind that the patient received tocilizumab 10 weeks after the last session of radiotherapy and the clinical improvement started to take place already after the second tocilizumab infusion, we feel that it would be an unlikely coincidence that the improvement of the TED was due to the late effect of the combined effect of iv steroids/radiotherapy rather than the beneficial effect of tocilizumab.

In this case, sight-threatening TED was manifested with reduction of visual acuity, uncontrolled intraocular pressure and deterioration of visual fields in a man who responded poorly to both a 3-day high dosage treatment with intravenous methylprednisolone and to a 12-week course of the same steroid as suggested by EUGOGO. This 12-week course of iv steroids is recommended for moderate-to-severe TED by EUGOGO but still we elected to administer the treatment to the patient in order to achieve a prolonged anti-inflammatory effect with the aim to reduce the volume of the extraocular muscles that compressed the optic nerves as confirmed on the orbital CT and the visual fields. We decided to proceed with tocilizumab as orbital decompression was rejected by the patient and gradual deterioration of the visual fields and high GAT-IOPs were recorded. Literature suggests that tocilizumab in moderate-to-severe TED reduces the soft tissue swelling, Clinical Activity Score and exophthalmos but this improvement does not translate into significant clinical improvement from the patients' point of view $(8,9)$. The decision to favor tocilizumab over other immunoregulatory modalities was based on the safety profile of tocilizumab, the supporting evidence from the international literature $(8,10)$ and the lack of evidence that tocilizumab is inferior to the other medical treatments.

Our patient had very congested orbits with the soft tissues impinging on the optic nerves. We also felt that the high IOPs were due to poor aqueous outflow secondary to episcleral venous congestion. The patient's response was impressive which reflected on the improvement of the eyelid and conjunctival swelling and redness, the improvement of the ocular motility, the reduction of the IOP (presumably due to the decrease of the episcleral venous pressure) and the ameliorated perimetric findings secondary to the reduction of the soft tissues bulk. Exophthalmometry readings did not change although the upper and lower lid retraction was minimally improved compared to the baseline measurements.

We believe that tocilizumab can have a positive impact on the aforementioned aspects of TED. However it is very unlikely that it could be used in the case of exposure keratopathy as it does not reduce the eyelid apertures (8). In the same manner dysthyroid optic neuropathy which can cause quick and profound loss of vision should not be treated with tocilizumab as its effects take days to weeks to develop. It should, though, be used in cases were the first line medication fails to offer adequate alleviation of signs and symptoms of moderate-to-severe TED.

Sy et al. (10) reported two cases of sight-threatening TED who received iv tocilizumab after orbital decompression. Tocilizumab improved the signs of TED although the previous decompressions did not have the desired effects. The anti-inflammatory effect of tocilizumab was also confirmed with orbital fat biopsies.

Research has shown that thyroidectomy may improve the signs of TED but this process takes months to develop $(11,12)$. In the case of our patient we did not have the time to wait for the possible beneficial effects of thyroidectomy. For this reason we started the patient on the tocilizumab treatment 6 weeks after the thyroidectomy. We believe that the improvement of symptoms was due to the tocilizumab treatment as it is was already observed from the second intravenous infusion and the time course by that time was too short for the effects of the thyroidectomy to manifest. The patient was euthyroid on tablets of levothyroxine after the removal of the thyroid gland.

As far as it concerns side effects, tocilizumab can cause hepatitis, opportunistic infections or flare ups of latent infections as it interferes with the immune response and development or exacerbation of cancer (8). Our patient developed a moderate thrombocytopenia which recovered after 3 weeks without intervention.

To the best of our knowledge this is the first case reported where tocilizumab was used in a patient with severe TED. Although these cases require treatment that evoke a quick response we felt the rate of the development of the optic neuropathy gave us the luxury of time to use a medication that takes longer to act and indeed the clinical response supported our decision. We would like though to stress that tocilizumab (as other immunoregulatory medication) is not a substitute for the recommended management of severe TED and its main indication is moderate-to-severe disease.

\section{CONCLUSIONS}

In the present article we describe a case of sight threatening thyroid eye disease which was treated with tocilizumab. This IL- 6 inhibitor plays a key role in reducing the swelling of the soft tissues in moderate-to-severe TED. Our patient benefited from this treatment as the extraocular muscles compressed the optic nerve causing symptoms and signs of dysthyroid optic neuropathy. The reduction of the soft tissue swelling led to an improvement of the optic nerve function that was maintained at the last follow up eight months after the last intravenous dose of tocilizumab.

\section{AUTHORS' STATEMENT OF CONSENT}

The authors have obtained written consent by the patient stating that the patient agrees to have the pictures attached to this paper published. 


\section{REFERENCES}

1. Barrio-Barrio J, Sabater LA, Bonet-Farriol E, Velázquez-Villoria A, Galofré JC. Graves' Ophthalmopathy: VISA versus EUGOGO Classification, Assessment, and Management. J Ophthalmol 2015; 2015: 249125.

2. Bartalena L. What to do for moderate-to-severe and active Graves' orbitopathy if glucocorticoids fail? Clin Endocrinol (Oxf) 2010; 73(2): 149-52.

3. Hamed Azzam S, Kang S, Salvi M, Ezra DG. Tocilizumab for thyroid eye disease. Cochrane Database Syst Rev 2018; 11: CD012984.

4. Bartalena L, Baldeschi L, Boboridis K, et al. The 2016 European Thyroid Association/European Group on Graves' Orbitopathy guidelines for the management of Graves' orbitopathy. Eur Thyroid J 2016; 5(1): $9-26$.

5. Strianese D, Rossi F. Interruption of autoimmunity for thyroid eye disease: B-cell and T-cell strategy. Eye (Lond) 2019; 33(2): 191-9.

6. Shams PN, Ma R, Pickles T, Rootman J, Dolman PJ. Reduced risk of compressive optic neuropathy using orbital radiotherapy in patients with active thyroid eye disease. Am J Ophthalmol 2014; 157(6): 1299-305

7. Marcocci C, Bartalena L, Bogazzi F, Bruno-Bossio G, Lepri A, Pinchera A. Orbital radiotheraphy combined with high-dose systemic gluco- corticoids for Graves' ophthalmopathy is more effective than orbital radiotherapy alone: results of a prospective study. J Endocrinol Invest 1991; 14: 853-60.

8. Perez-Moreiras JV, Gomez-Reino JJ, Maneiro JR, et al. Tocilizumab in Graves Orbitopathy Study Group. Efficacy of Tocilizumab in Patients With Moderate-to-Severe Corticosteroid-Resistant Graves Orbitopathy: A Randomized Clinical Trial. Am J Ophthalmol 2018; 195 : 181-90.

9. Maldiney T, Deschasse C, Bielefeld P. Tocilizumab for the Management of Corticosteroid-Resistant Mild to Severe Graves' Ophthalmopathy, a Report of Three Cases. Ocul Immunol Inflamm 2020; 28(2): 281-4.

10. Erdoğan MF, Demir O, Ersoy RU, et al. Comparison of Early Total Thyroidectomy with Antithyroid Treatment in Patients with Moderate-Severe Graves' Orbitopathy: A Randomized Prospective Trial. Eur Thyroid J 2016; 5(2): 106-11.

11. Sy A, Eliasieh K, Silkiss RZ. Clinical Response to Tocilizumab in Severe Thyroid Eye Disease. Ophthalmic Plast Reconstr Surg 2017; 33(3): e55-e57.

12. Weber KJ, Solorzano CC, Lee JK, Gaffud MJ, Prinz RA. Thyroidectomy remains an effective treatment option for Graves' disease. Am J Surg 2006; 191: 400-5. 